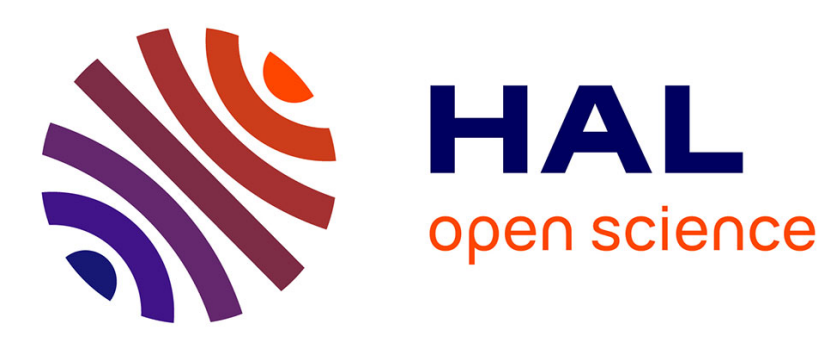

\title{
Sur la date et l'auteur du remaniement du Roman de la Rose par Gui de Mori
}

\author{
Andrea Valentini
}

\section{To cite this version:}

Andrea Valentini. Sur la date et l'auteur du remaniement du Roman de la Rose par Gui de Mori. Romania (Paris), 2006, 124 (495), pp.361-377. 10.3406/roma.2006.6863 . hal-03371714

\section{HAL Id: hal-03371714 https://hal.science/hal-03371714}

Submitted on 8 Oct 2021

HAL is a multi-disciplinary open access archive for the deposit and dissemination of scientific research documents, whether they are published or not. The documents may come from teaching and research institutions in France or abroad, or from public or private research centers.
L'archive ouverte pluridisciplinaire HAL, est destinée au dépôt et à la diffusion de documents scientifiques de niveau recherche, publiés ou non, émanant des établissements d'enseignement et de recherche français ou étrangers, des laboratoires publics ou privés. 


\title{
SUR LA DATE \\ ET L'AUTEUR DU REMANIEMENT \\ DU ROMAN DE LA ROSE PAR GUI DE MORI
}

1. L La date

\author{
En l'an de l'incarnation \\ Jhesucrist par duplation \\ De .VI ${ }^{c}$. et .V. et .XL., \\ Le jeudi devant çou c'on chante \\ Resurrexi, fu terminés \\ Cis roumans et ensi finés \\ Com maistres Guillaumes le finne... \\ (Ms. Tournai, Bibl. de la Ville, $101=T_{0 u}{ }^{~}$, f. 41 b)
}

La partie de Jean de Meun du Roman de la Rose remanié par Gui de Mori est introduite par ces vers dans le seul manuscrit existant du remaniement complet (sauf quelques feuillets arrachés ${ }^{2}$ ). Déjà Ernest Langlois, dans le premier article consacré au sujet, affirmait que le travail aurait été accompli en $1290:$ " par duplation [duplication] / De .VIc et .V. et .XL. » $=645 \times 2$, en précisant : « peut-être en 1291, si le rimeur faisait commencer l'année à Pâques ; mais c'est très peu probable " ${ }^{3}$. En réalité,

1. Sauf indication contraire, nous utilisons les sigles introduits par E. Langlois, Les Manuscrits du 'Roman de la Rose'. Description et classement, Lille-Paris, 1910, p. 238-240 [Travaux et mémoires de l'Université de Lille, n. s., 7].

2. Les f. $32,37,114-116,132,162,168$ ont été remplacés au XIV ${ }^{\mathrm{e}}$ siècle; les f. 118-119 ont été remplacés au $\mathrm{xv}^{\mathrm{e}}$ siècle ; il manque un feuillet entre les f. 37-38, 91-92, 95-96, 119-120, 132-133, 168-169, deux entre les f. 93-94, 134-135.

3. E. Langlois, "Gui de Mori et le Roman de la Rose ", dans Bibliothèque de l'École des Chartes, t. 68 (1907), p. 249-271, p. 249 et n. 4. 
le style de Pâques était suivi de préférence dans l'Artois, lieu d'origine de l'auteur selon Langlois (voir ci-dessous), ainsi que dans le Hainaut français ${ }^{4}$, mais il est possible qu'il faille entendre 1290 pour une autre raison, que nous expliquerons ci-dessous.

Cette date est corroborée par une autre, qu'on lit dans une interpolation insérée dans le passage où le dieu d'amour prophétise l'existence de Guillaume de Lorris et de Jean de Meun ; le remanieur ajoute la prophétie de son existence :

\author{
On ora de lui mention \\ En l'an de l'incarnation \\ Mil et .II' . et quatrevins \\ Et dis, desci j'en sui devins ; \\ Guis de Moiri avra a non... \\ ( $T o u$, f. 96 c, après le v. $10596^{5}$ )
}

Cette date, 1290, a été acceptée par tous les auteurs qui ont accompli des études approfondies sur Gui de Mori ${ }^{6}$; assez récemment, pourtant, Lori Walters a proposé de lire les indications chronologiques de façon différente. En ce qui concerne la première datation, elle écrit : « if the preposition applies solely to ".VIc.", and only 600 is multiplied by 2 , the answer becomes 1245 " $^{7}$ : ce serait en cette année que le remanieur aurait terminé la révision de la première partie du poème. On serait là en présence d'un fait d'une extraordinaire importance, parce qu'on aurait la confirmation

4. Cf. A. Cappelli, Cronologia, cronografia e calendario perpetui, Milan, 1987 p. 12-13. L'origine picarde de l'auteur était déjà affirmée par E. Langlois, art. cit., p. 257 ; la présence de quelques traces d'une scripta picarde même dans les témoins qui utilisent une koiné linguistique, ainsi que l'analyse des rimes, confirment l'affirmation de Langlois. Nous publierons bientôt l'édition des interpolations de Gui de Mori, avec une analyse de sa langue dans la collection Anciens auteurs belges de l'Académie royale de Belgique.

5. Nous suivons la numérotation de l'édition de F. Lecoy (Guillaume de Lorris et Jean de Meun 'Le Roman de la Rose', 3 vol., Paris, 1965-1970 [CFMA, 92, 95 et 98]). Pour la numérotation de l'édition d'E. Langlois (Guillaume de Lorris et Jean de Meun 'Le Roman de la Rose', 5 vol., Paris, 1914-1924 [SATF]) dans la partie de Jean de Meun, ajouter trente unités; pour la partie de Guillaume de Lorris, nous indiquerons les correspondances.

6. M.-R. Jung, "Gui de Mori et Guillaume de Lorris ", dans Vox Romanica, t. 27 (1968), p. 106-137, à la p. 106 ; D. Hult, « Gui de Mori, lecteur médiéval », dans Incidences, n. s., t. 5 (1981), p. 53-70, à la p. 60 ; S. Huot, The 'Romance of the Rose' and its Medieval Readers. Interpretation, Reception, Manuscript Transmission, Cambridge, 1993 [Cambridge Studies in Medieval Literature, 16], à la p. 85.

7. L. Walters, "Who was Gui de Mori ? ", dans "Riens ne m'est seur que la chose incertaine ". Études sur l'art d'écrire au Moyen Âge offertes à Eric Hicks, J.-C. Mühlethaler et D. Billotte éd., Genève, 2001, p. 133-144 [Travaux des Universités suisses, 9], à la p. 136. 
que la première partie du Roman de la Rose fut écrite et circulait déjà avant 1245 , ce qui n'est qu'une hypothèse basée sur les vers célèbres de Jean de Meun ${ }^{8}$. Pour ce qui relève de la deuxième datation, $L$. Walters affirme que " "dis" is not part of the date, but rather the first person singular, indicative tense, of the verbe "dire", stated with omission of the subject $"{ }^{9}$; il faudrait donc entendre 1280 : à cette date, Gui de Mori aurait terminé le remaniement de la deuxième partie.

Les données de la tradition manuscrite semblent confirmer la première datation retcnuc. Dans le manuscrit Paris, BNF, n.a.f. 28047, appelé Ter par Marc-René Jung ${ }^{10}$, on lit : "par dupplation / De .VI ${ }^{c}$. de .V. (et) .XL." (f. 34a) ${ }^{11}$, où la préposition précède aussi "V et XL" ${ }^{12}$. La relation entre « duplation » et tous les chifres qui suivent aussi dans Tou, par ailleurs, ne surprend pas, puisque en ancien français la conjonction " et » était souvent utilisée pour former les nombres par addition ${ }^{13}$; l'interversion des chiffres («.V. et .XL. ») n'est pas étonnante non plus en ancien français dans un texte en vers ${ }^{14}$.

8. «...car quant Guillaumes cessera, / Jehans le continuera, / enprés sa mort, que je ne mante, / anz trespassez plus de .XL.... » (v. 10557-10560).

9. "Who was Gui de Mori ? ", art. cit., p. 135. Dans ce cas, il faudrait placer une virgule après " quatrevins ", et remplacer le point-virgule après « devins " par une virgule ou par deux-points.

10. "Gui de Mori et Guillaume de Lorris », art. cit., p. 106. Le manuscrit appartint à l'abbé Campion de Tersan, archidiacre de Lectoure, mort en 1819.

11. Ce manuscrit, qui était auparavant en main privée et qui a suscité pendant longtemps la curiosité des médiévistes et notamment des spécialistes du Roman de la Rose, a enfin été acheté par la BNF lors d'une vente aux enchères le 7 avril 2006 (Une bibliothèque littéraire. Livres et manuscrits du $X I V^{v}$ au $X X^{v}$ siècle, par Pierre Bergé et Associés, Paris, Drouot Richelieu, $\mathrm{n}^{\circ}$ 9, p. 14-16 du catalogue). Nous en avons rédigé une notice, qui paraîtra dans Romance Philology au printemps 2007. Méon avait été le dernier savant à pouvoir consulter ce codex et avait transcrit le prologue de la partie de Jean de Meun par Gui de Mori dans l'introduction de son édition du Roman de la Rose (4 vol., Paris, 1814, t. 1, p. IX-X).

12. Walters, qui cite à partir de Méon, lit " par dupplication / de VIc et de V et $\mathrm{XL}$ », mais il s'agit d'une mauvaise lecture de la transcription, qui rend le vers hypermètre ("Who was Gui de Mori ? », art. cit., p. 136, n. 12).

13. Voir, par exemple, G. Moignet, Grammaire de l'ancien français. Morphologie, syntaxe, Paris, $1976^{2}$, p. 50.

14. Voir, entre autres, Histoire de l' Abbaye de Fécamp, v. 4561-4563 : « Ot mil anz et nuef et nonante / Ke Dex de la Virge waillante / Avoit pris incarnation » (cité par A. Tobler-E. Lommatzsch, Altfranzösisches Wörterbuch, 11 vol., BerlinWiesbaden-Stuttgart, 1925-2002, t. 4, col. 1361, d'après A. Långfors éd., Histoire de l'Abbaye de Fécamp en vers français du XIII ${ }^{e}$ siècle, Helsinki, 1928 [Annales Academiae Scientiarum Fennicae, XXII 1]); Rutebeuf, La Vie de sainte Elysabel, v. 122-124: " En l'an de l'incarnacion / Mil et .II. cens et quatre et trente, / Si com l'escriture le chante » (M. Zink éd., Rutebeuf 'CEuvres complètes', Paris, 2001 ${ }^{2}$ 
Pour ce qui est de la deuxième date, dans le ms. Paris, BNF, fr. $797=$ Mor ${ }^{15}$, on lit «X » à la place de « dis » (f. 90c). Mais la raison majeure pour laquelle l'interprétation de $\mathrm{L}$. Walters est difficile à retenir est le fait que la première personne du singulier de l'indicatif présent du verbe " dire » est, au XII ${ }^{\mathrm{e}}$ siècle, " di » et non pas « dis » 16 : cette dernière forme, en effet, n'est jamais employée dans Tou, qui est daté de 1330 (voir ci-dessous), alors qu'on trouve souvent « di » (f. 7a, 24b, 29d, 30b, etc.). En outre, la syntaxe non plus ne serait pas totalement convaincante, si l'on entendait « dis » comme première personne du singulier du verbe " dire » avec omission du sujet, tout en n'étant pas complètement inacceptable. Dans les interpolations ajoutées par Gui de Mori, on rencontre ailleurs des vers qui commencent par la conjonction « et » suivie d'une forme du verbe dire sans sujet, mais il s'agit toujours de propositions coordonnées à d'autres où le sujet est le même et clairement identifiable - ce qui ne se vérifie pas dans le passage qui nous intéresse - comme dans l'extrait suivant :

L'escripture pas ne maudist Jonece, ançois le resbaudist, Et dist que c'est moult boine cose Quant jones hom son col souspose...

(Tou, f. 46d, après le v. 4514) ${ }^{17}$

[Lettres Gothiques]) ; et le Roman de la Rose même, v. 11766-11767 : « en l'an de l'incarnacion /.M. e.II.C. .V. e.L." ".

15. Nous adoptons le sigle introduit par S. Huot, The 'Romance of the Rose' and its Medieval Readers, art. cit., p. XV.

16. Je remercie Mme Geneviève Hasenohr d'avoir porté mon attention sur ce point. Voir, entre autres, P. Fouché, Le Verbe français. Étude morphologique, Paris, 1967², p. 119-120 [Tradition de l'humanisme, 4] ; G. Raynaud de Lage, Introduction à l'ancien français, nouv. éd. par G. Hasenohr, Paris, $1993^{2}$, p. 141 ; « après voyelle, [...] l'évolution [vers l'extension de la désinence $-s$ ] est beaucoup plus lente [par rapport aux verbes dont les radicaux se terminent par une consonne], sauf pour la première personne du singulier de estre [...]. Plusieurs des textes que nous avons examinés connaissent les deux formes pour dire : di / dis [...] ; mais dans d'autres textes, et jusqu'à l'extrême fin $\mathrm{du}_{\mathrm{xv}^{\mathrm{e}}}$ siècle, nous ne rencontrons que je di» (C. Marchello-Nizia, Histoire de la langue française aux $X I V^{e}$ et $X V^{\prime \prime}$ siècles, Paris, 1979, p. 202).

17. Cf. également : « Li sages son fil amoneste / Et dist : 'Biaus fieus, ne manifeste / Ton coer a cascune personne... " (Tou, f. 53a, après le v. 4677); "Aucun les ensengnemens prisent / De proiier, aucun les desprisent / Et dient, a lor enscïent, / Que proiiers n'i vaut mais nïent 》 (Tou, f. 80a, après le v. 8320) ; "S'il avient c'aucuns a lui viengne / Qui de li sa parole tiengne / Et dic k'il pcrt son cstuide... » ( $T o u$, f. 84a, après le v. 9712), etc. 
En fait, dans sa thèse, Torsten Franzén affirmait : " quand la conjonction et relie deux propositions qui n'ont pas le même sujet, il est naturel que le pronom sujet soit exprimé après $e t$ », bien qu'il ajoute qu' « on rencontre pourtant des cas où le pronom personnel est omis $\gg 18$.

Nous pensons donc qu'il faut retenir la date de 1290 , d'autant que le passage en question est postérieur au premier passage cité, où il faudrait postuler une faute dans le texte pour ne pas lire 1290 , à moins de comprendre l'année comme s'il s'agissait de 1245 , ce qui semble très peu probable.

En ce qui concerne les rapports entre les deux passages cités, on remarque que, dans le premier, l'auteur affirme écrire « le jeudi devant çou qu'on chante / Ressurrexi », c'est-à-dire le jeudi saint : or, si le remanieur suivait le style de Pâques, à la rigueur il devait se trouver en 1290 quand il écrivit la première datation et en 1291 quand il écrivit la deuxième, parce qu'il paraît peu probable qu'il ait pu composer les milliers de vers présents entre le premier et le deuxième passage en deux ou trois jours; mais il aurait bien pu avoir écrit son prologue à la partie de Jean de Meun après coup, ou, même en écrivant (ou en imaginant d'écrire ?) le jeudi saint, utiliser déjà la date de la nouvelle année ${ }^{19}$.

Il existe un autre élément qui permet de dater le remaniement autour de l'année 1290 plutôt que de l'année 1280. Dans l'œuvre, en effet, on cite la bulle papale $A d$ fructus uberes, émise par le pape Martin IV en décembre 1281 ou en janvier $1282^{20}$; c'est Faux Semblant qui parle :

18. Études sur la syntaxe des pronoms personnels sujets en ancien français, Uppsala, 1939, p. 60.

19. Sur le conventionnalisme des datations au Moyen Âge, cf. M. Zink, La Subjectivité littéraire. Autour du siècle de saint Louis, Paris, 1985, p. 81-125. Si, nous aussi, voulions nous plier à ce jeu des dates et si nous considérions que la date est à prendre pour 1290, pour les raisons que nous venons d'expliquer, même si le remanieur pouvait utiliser le style de Pâques dans la vie courante, nous pourrions fixer avec précision le jour auquel Gui de Mori affirme avoir écrit : Pâques étant le 2 avril en 1290, le prologue aurait été écrit le 30 mars.

20. «Anno 1281. die 13. Decembr. al. 1282. die 10. Januarii » : cf. J. H. Sbaralea, Bullarium franciscanum Romanorum pontificum constitutiones, epistolas ac diplomata continens tribus ordinibus Minorum, Clarissarum et Pcenitentium a Seraphico Patriarcha Sancto Francisco institutis concessa ab illorum exordio ad nostra usque tempora, t. 3, Rome, 1765 , p. 480c. Walters écrit que « Gui makes no mention of Martin IV's bull, Ad fructus uberes ( (L. Walters, "Gui de Mori's Rewriting of Faux Semblant in the Tournai Roman de la Rose », dans The Medieval Opus. Imitation, Rewriting, and Transmission in the French Tradition, D. Kelly éd., Amsterdam-Atlanta, 1996, p. 261-276 [Faux titre. Études de langue et littérature françaises, 116], à la p. 275). 
Mais li papes, qui tout souspoie 21, Vit assés tost que je pensoie

Et me feri d'une retraite

Par une retenue faite

En le fin de ce previliege,

Qui tient ausci com en .I. piege

Les sougis en tel obligance

Qu'il doivent, sans nule doutance

Ne sans querre barre ne tour,

A lors curés avoir retour

Selonc le general concille,

Et c'a chou les devons sans guille

Diligentement enorter.

(...)

Car une foys l'an au mains doivent

Tout a chelui dont il reçoivent

Sacrement et conmunion

Faire entiere comfiession.

(Tou, f. $106 \mathrm{~cd}$, après le v. 11568)

Ces vers sont à comparer avec la partie finale de la bulle citée (" le fin de ce previliege $»$ : dans la bulle, le pape autorise les ordres mendiants à prêcher et à confesser) :

Volumus autem, quod hi, qui Fratribus confitebuntur eisdem, suis Parochialibus Presbyteris confiteri saltem semel in anno, prout Generale Concilium statuit, teneantur. Quodque iidem Fratres eosdem ad hoc diligenter, et efficaciter secundum datam eis gratiam a Domino exhortentur. Nulli ergo omnino hominum liceat hanc paginam Nostræ Concessionis, et Inhibitionis infringere, uel ei ausu temerario contraire 22

On remarque en particulier les correspondances entre "Selonc le general concille » et " prout Generale Concilium statuit ", " a chou les devons (...) / Diligentement enorter » et " iidem Fratres eosdem ad hoc diligenter (...) exhortentur », « une foys l'an au mains » et « saltem semel in anno $\gg$.

Ce passage suit celui où on lit la deuxième datation, et l'on pourrait imaginer que le remanieur a fait circuler son ouvrage sans avoir revu les indications chronologiques (au cas où il faudrait lire 1280); mais si l'on considère cette citation avec les éléments offerts par la tradition

21. "Souspoie » est la leçon de Ter (f. 108c), confirmée par le ms. Copenhague, Bibl. Royale, Gl. Kgl. Sam. $20614^{\circ}=H e$, qui a "suppoie " (f. 92a), variante graphique de « souspoie »; Tou a « souspoise », qui fausse la rime.

22. J. II. Sbaralea, Bullarium franciscanum Romanorum pontificum constitutiones, epistolas ac diplomata continens, éd. cit., p. $480 \mathrm{~cd}$. 
manuscrite, par l'analyse syntaxique et par la grammaire historique, nous croyons qu'il faut préférer la datation traditionnelle, qui date le remaniement de 1290.

\section{2. - L'énigme du nom de l'auteur}

Ainsi, l'identification de Gui de Mori avec Guibert de Tournai, proposée par Lucien Fourez ${ }^{23}$ et reprise par Lori Walters ${ }^{24}$, devient impossible : ce maître franciscain de l'Université de Paris, conseiller de saint Louis et auteur de quelques traités en latin, né apparemment vers $1200^{25}$, mourut très vraisemblablement le 7 octobre 1284, comme l'a démontré le Père Heysse ${ }^{26}$. Cependant, cette identification était ingénieuse, parce qu'elle paraît résoudre une sorte d'énigme qu'on lit dans le même prologue de la partie de Jean de Meun évoqué ci-dessus (le vers qui nous intéresse est en italique) :
Et se de mon non voet avoir
Aucuns aucune congnissance,
Ne l'en ferai ja demoustrance
Autrement fors que par mos teus,
C'on entre par moy es osteus :
De plus je ne descouverroie,
Moy ne mon sornon ne vorroie
Rimer ne par rime retraire.

(Tou, f. $41 b)$

Or, Guibert de Tournai appartenait à la noble famille tournaisienne des Morielporte d'As-Piés ${ }^{27}$, et « on entre dans un hôtel par la porte et au moyen des pieds ", comme le faisait remarquer Fourez ${ }^{28}$.

Le remanieur révèle apparemment son nom plus tard dans le poème, dans la prophétie du dieu d'amour :

23. "Connaît-on le troisième auteur du Roman de la Rose de Tournai ? ", dans Tournay. Reconstruction et avenir, t. 55-56 (juillet-août 1950), p. 4.

24. "Who was Gui de Mori? ", art. cit.

25. Pour un aperçu de la vie et des ouvres de Guibert de Tournai, cf. B. d'Amsterdam, "Guibert de Tournai », dans Dictionnaire de spiritualité, t. 7, Paris, 1967, p. 1139-1146.

26. A. Heysse, "De die obitus Fr. Gilberti Tornacensis ", dans Archivum franciscanum historicum, t. 26 (1933), p. 558-559.

27. B. d'Amsterdam, " Guibert de Tournai », art. cit., p. 1139.

28. "Connaît-on le troisième auteur du Roman de la Rose de Tournai ?", art. cit., p. 4c. 
Guis de Moiri avra a non,

Mais il n'ert pas de tel regnon

Com cis Jehans ne chil Guillaumes ...

(Tou, f. 96c, après le v. 10596)

À vrai dire, on trouve déjà le nom Gui de Mori dans les rubriques qu'on lit dans Tou au début du manuscrit ( «...i sont les adictions dant Guy de Mori », f. 1a, et « Chi conmence li prologes que dant Gui de Mori fist sur le Livre de la Rose ", f. 5r) et dans Ter (seulement le prénom) après les vers mêmes où on lit l'énigme ( «Aprés ce que je Guis devant dis euc ce premier livre aussi fait comme chi deseseure [lire deseure] est contenu, vint entre mes mains li livres maistres Jehan de Meun..." ", f. $34 \mathrm{bc})^{29}$, mais il s'agit là de parties en prose, où la fiction énigmatique n'avait peut-être pas sa place, d'autant que l'auteur écrit qu'il ne veut pas «rimer » son nom et son surnom ${ }^{30}$. Mis à part ces passages en prose, l'auteur a-t-il décidé de révéler son nom à un certain moment, un moment important, celui de la prophétie du dieu d'amour, qui est aussi le seul endroit où Jean de Meun se nomme lui-même, avec son prédécesseur ? Ou faut-il penser, comme le suggérait Fourez, que sous l'énigme ne se cache pas le nom " Gui de Mori », qui ne serait qu'un pseudonyme, mais une autre solution? L'auteur appartiendrait-il lui aussi à la famille des Morielporte, comme Guibert de Tournai, sans qu'il faille nécessairement identifier les deux personnages, ou à la limite à la famille des Le Porte ${ }^{31}$, elle aussi de Tournai ?

Pendant quelque temps, nous avons pensé que cette solution pouvait être la bonne et que l'auteur devait être quelquc Guillaume ou Guibert de Morielporte ou Le Porte, même si nous n'avons trouvé aucune trace d'un membre de cette famille qui puisse être identifié avec le remanieur. Bien que les somptueuses archives de la ville de Tournai aient été détruites lors de la seconde guerre mondiale ${ }^{32}$, l'existence de personnages nommés

29. Ce « devant dis » doit se référer à une rubrique insérée dans la partie de Guillaume de Lorris : mais, comme nous le démontrerons dans l'article cité à la n. 11, dans Ter la première partie ne contient pas de rubriques et est copiée d'après un manuscrit non remanié.

30. Nous avons quelques doutes sur la justesse de la leçon «par rime » de Tou: Ter donne "par apiert ", qui évite une répétition. Nous ajoutons qu'il n'est pas certain, même si cela reste probable, que Gui de Mori soit l'auteur des cent vingt-six rubriques de Tou.

31. Déjà Méon interprétait de cette façon l'énigme : "il paroît que cet auteur se nommoit la Porte, et je n'ai rien trouvé sur lui " (Guillaume de Lorris et Jehan de Meung 'Le Roman de la Rose', éd. cit., t. 1, p. X).

32. Sur la richesse de ces archives, cf. $\mathrm{P}$. Ruelle, Chartes en langue française antérieures à 1271 conservées dans la province de Hainaut, Paris, 1984, p. XIX [Documents linguistiques de la Belgique romane, 1]. 
Guibert et Guillaume appartenant à ces familles est attestée par quelques transcriptions d'actes des XIII ${ }^{\mathrm{e}}$ et $\mathrm{XIV}^{\mathrm{e}}$ siècles, ce qui ne surprend pas ${ }^{33}$.

Mais est-il sûr qu'il faille nécessairement chercher notre auteur à Tournai ? Il est vrai que le manuscrit principal qui témoigne de ce remaniement, Tou, a été produit pour un membre de la famille tournaisienne des Pourrès en $1330^{34}$; mais, malgré son extrême correction, rien ne prouve qu'il soit une copie contrôlée par Gui de Mori et non pas une copie fort exacte d'un original perdu ${ }^{35}$. En outre, quelqu'un qui travaillait pour un Tournaisien n'était pas nécessairement originaire de Tournai.

Nous nous demandons maintenant si la solution n'est pas plus facile qu'on pourrait le croire, et si la devinette en question ne se réfère pas tout simplement au prologue dans lequel elle est insérée, et non pas à l'œuvre entière, d'autant plus que dans Ter, dans les vers cités ci-dessus, on lit « $\mathrm{Ne}$ l'en ferai or demonstrance » pour « Ne l'en ferai ja demoustrance » et « De plus ore ne descouverroie $[+1=$ or ?]» pour «De plus je ne descouverroie » : pas maintenant ${ }^{36}$, mais plus tard oui.

33. Un Guibert de Morielporte fut échevin à Tournai entre 1223 et 1236 (ou s'agit-il de deux personnages ?) : cf. A. d'Herbomez, « Chartes françaises du Tournaisis (1207-1292) ", dans Mémoires de la Société historique et littéraire de Tournai, t. 17 (1882), p. 1-62, aux p. 11, 12,13,16,18,19-20, 22, 26 et 30, ainsi que P. Ruelle, op. cit., p. 3-4. Dans l'ouvrage de ce dernier, on constate également qu'un Guibert et un Guillaume de le Porte vivaient en 1266 (p. 116-117), peut-être les mêmes dont rendent compte $A$. de la Grange, "Choix de testaments tournaisiens antérieurs au $\mathrm{XVI}^{\mathrm{e}}$ siècle ", dans Annales de la Société historique et archéologique de Tournai, n. s., t. 2 (1897), p. 5-365, à la p. 29, et A. Hocquet, « Table des testaments, comptes de tutelle et d'exécution testamentaire reposant aux Archives de Tournai ", Ibid., n. s., t. 6 (1901), p. 281-299, à la p. 288. En 1349, vivait un «Deleporte Wille" (A. Hocquet, "Table des testaments, comptes de tutelle et d'exécution testamentaire reposant aux Archives de Tournai », Ibid., n. s., t. 7 (1902), p. 81-161, à la p. 103), peut-être le même dont La Grange atteste l'existence en 1365 (art. cit., p. 89).

34. L'écu des Pourrès a été reconnu dans celui qui est reproduit à côté d'un personnage agenouillé à droite du dieu d'amour dans le bas de page du f. $5 \mathrm{r}$ par A. Kuhn, «Die Illustration der Handschriften des Rosenromans », dans Jahrbuch der Kunsthistorischen Sammlungen des allerhöchsten Kaiserhauses, t. 31 (1912), p. 1-66, à la p. 24, n. 2. On lit la date dans l'explicit : « Escris fu l'an .mil. et .CCC. / Et .XXX. ", f. 171a.

35. Cf. une de nos précédentes contributions : A. Valentini, « Le remaniement de Gui de Mori et sa tradition manuscrite », dans De la Rose. Texte, image, fortune, C. Bel et H. Braet éd., Louvain, 2006, p. 299-320 [Synthema, 3], aux p. 304305.

36. Par ailleurs, bien que la signification la plus répandue de « ja » dans une phrase négative ( $\mathrm{Ne}(.$.$) ja » de Tou) soit celle qui correspond au français moderne$ "ne...jamais", selon W. von Wartburg, Französisches Etymologisches Wörterbuch, 25 vol., Tübingen-Bcrlin-Bâlc-Nancy, 1922-2002, t. 5, p. 26b, il existe des cas où « ne ... ja » pouvait signifier simplement « ne ... pas ». 
Quelle pourrait être alors la solution de la devinette ? Selon David Hult, il faudrait penser au mot "guichet», d'autant que «par cette petite pirouette le nom de notre auteur / copiste devient, pour l'Amant, et en même temps pour le lecteur, le portail d'entrée du monde allégorique du jardin de Deduit ${ }^{37}$. Même si la référence au texte même du roman reste probable, nous croyons que la devinette peut être résolue de façon encore plus simple : puisque en picard le $w$-germanique initial était souvent conservé ${ }^{38}$, un mot comme Guis (cas sujet, qui est le seul que l'on retrouve dans les vers du remaniement, à deux reprises, dans le passage cité ci-dessus et peu après dans la même interpolation), parfois noté « Wis » ${ }^{39}$, pouvait se confondre avec « uis » < osTIUM, lui-même écrit « wis » parfois dans la scripta picarde ${ }^{40}$ (mot qui, selon Noël Dupire, serait à la base justement du français « guichet " ${ }^{41}$ ).

De toute façon, sauf découvertes inespérées, il semble que notre remanieur doive rester parmi ces auteurs connus uniquement par leur nom et par leur œuvre, nombreux dans la littérature médiévale : il est possible que Gui de Mori soit un pseudonyme, mais nous pensons que, même si c'est le cas, l'auteur se référait à ce nom dans la devinette. Mori serait-il donc un toponyme ? Comme le faisait remarquer Langlois, il existe un village du nom de Mory dans le domaine dialectal picard, actuellement dans le département du Pas-de-Calais, près d'Arras ${ }^{42}$ : nous ajoutons que le nom ancien de ce village était Moiri ${ }^{43}$, qui est la forme qu'on retrouve dans Tou (voir ci-dessus), ainsi que dans Ter, au f. $98 \mathrm{~b}$, et dans $M o r$, au f. $90 \mathrm{c}^{44}$.

37. " Gui de Mori, lecteur médiéval », art. cit., p. 65.

38. Ch.-Th. Gossen, Grammaire de l'ancien picard, Paris, 1970 [Bibl. française et romane. Série $A$ : manuels et études linguistiques, 19], p. 108-109.

39. Ibid., p. 108.

40. Cf. W. von Wartburg, FEW, op. cit., t. 7, p. 437a.

41. Cf. N. Dupire, «Alternances phonétiques en picard», dans Rom., t. 53 (1927), p. 151-176, aux p. 167-172. Du même avis, K. Baldinger, Dictionnaire étymologique de l'ancien français, G 9-10, Tübingen, 1995, col. 1599-1600, et F. Möhren, "'Guai victis !'. Le problème du gu initial roman ", dans Medioevo Romanzo, t. 24 (2000), p. 5-81, à la p. 72.

42. E. Langlois, « Gui de Mori et le Roman de la Rose », art. cit., p. 257.

43. Voir Dictionnaire topographique du département du Pas-de-Calais, comprenant les noms de lieu anciens et modernes, rédigé par le comte de Loisne, Paris, 1907, p. $265 \mathrm{~b}$.

44. Nous ferons simplement remarquer que notre auteur s'est peut-être amusé à suggérer son nom dans le prologue cité, où il affirme ne pas vouloir se nommer : en effet, on peut lire le toponyme, si c'en est un, dans le commencement des trois vers suivants : " $D \epsilon$ plus je ne descouverroie, / Moy ne mon sornon ne vorroie / Rimer ne par rime retraire $»$. 


\section{3. - La personnalité et le statut social de Gui de Mori}

Faute de renseignements externes, il faut chercher des traces de la personnalité du remanieur dans le texte même. Dans un prologue général qu'on ne trouve que dans Tou, on lit :

Et cil ki sont de no couvent

M'ont requis et proié souvent

C'un petit m'entente aploiasce

A ce c'aucun dit retrouvaisce

D'Amours et de ses dous conmans ...

(f. $5 b c)$

À partir de ces vers, Huot écrit : « it was probably for his fellow clerics that Gui prepared his version of the Rose " ${ }^{45}$, bien qu'elle traduise couvent par " convent / fellowship ", où le deuxième mot est plus indéterminé que le premier. En fait, couvent peut bien indiquer une confrérie de religieux, mais signifie aussi "compagnie, assemblée » 46 .

Dans l'interpolation où l'auteur révèle son nom, on lit (c'est le dieu d'amour qui parle de lui) :

Mais il ert en tele prison

Qu'il ne poroit sans mesprison

De lui les conmans maintenir

Qu'il convient les sergans [d'Amour] tenir.

Mais conment que il soit loiiés,

Tous dis ert ses coers aploiiés

A tous nos amis conseillier :

En lui avra bon conseillier.

(Tou, f. 96c)

Il faut voir là une allusion à la situation de moine ou, du moins, au vœu de chasteté 47 : dans d'autres œuvres médiévales, des expressions similaires ont cette signification ${ }^{48}$.

45. The 'Romance of the Rose' and its Medieval Readers, op. cit., p. 90.

46. Cf. A. Tobler-E. Lommatzsch, Altfranzösisches Wörterbuch, op. cit., t. 2, col. 989 .

47. Comme le disait déjà $\mathrm{S}$. Huot, The 'Romance of the Rose' and its Medieval Readers, op. cit., p. 90.

48. Nous pensons au début des Vers de la mort d'Hélinand de Froidmont: " Morz, qui m'as mis muer en mue / En cele estuve o li cors sue / Ce qu'il fist el siecle d'outrage... » (F. Wulff et $\mathrm{E}$. Walberg éd., Les Vers de la mort par Hélinant, moine de Froidmont, Paris, 1905 [SATF], v. 1-3), où la « mue » (cage, prison) représente le monastère même et métaphoriquement la condition de moine. Nous pensons encore au passage suivant, qu'on lit dans le prologue des Quinze joies de mariage : 
Gui de Mori a-t-il participé aussi à une vie de cour ? Dans la même interpolation, on lit des allusions à un monde fait d'envies, d'hypocrisies et de flatteries à l'égard des puissants :

Mais Fortune li [à Gui de Mori] ert contraire

Lonc tamps et si vil le tenra

C'a paines a honnour venra,

Male Bouce et sa compaignie

Li feront mainte vilonnie

En tant que de lui mesdiront

Et moult a souffrir li feront.

(...)

Il pora bien par lui savoir

Qu'en biau servir convient avoir

Eür, et iert auques seürs

K'a son tamps vaura mieus eürs

Que sens ne bontés ne biautés

Ne verités ne loiautés :

Verités iert si decheüe

Et flaterie si creüe

Que nus n'ara honnour ne pris,

S'il n'est de flaterie apris.

(Tou, f. 96d)

On peut lire une critique analogue de l'« ypocrisie », de la « flaterie » et des « losengeor », mêlée plus particulièrement à une critique de l'absence de reconnaissance des personnes qui atteignent des postes de pouvoir, dans une autre interpolation, qui suit le v. 4834 (à lire dans Tou aux f. $47 d$ 48a).

Mais ces thèmes peuvent bien être des expédients rhétoriques ; comme il est bien connu, l'autobiographie du Moyen Âge ne peut être exactement assimilée à ce que nous entendons aujourd'hui par ce concept. Si l'on devait croire sans contredit aux allusions de Gui de Mori, on remarquerait que des propos comme ceux qui suivent ne sont pas vraiment dignes de la morale d'un moine, telle qu'on pourrait se l'imaginer :

Mais de ce fera a blamer,

Que ja ne vorra bien amer :

"...moy aussi, pensant et considerant le fait de mariage ou je ne fu oncques, pour ce qu'il a pleu a Dieu me mectre en aultre servage hors de franchise que je ne puis plus recouvrer, ay advisé que en mariage a .XV. serimonies... " (J. Rychner éd., Les. $X V$. joies de mariage, Genève, 1963 [TLF, 100], p. 4); l'auteur des Quinze joies, en revanche, n'appartenait pas au clergé régulier, selon son éditeur, et donc l'« aultre servage " ne serait que l'état de clerc (pourtant, dans son édition, Rychner justifie son opinion seulement par l'hostilité de l'auteur vis-à-vis des cordeliers et des jacobins : ibid., p. 4 et n. 101). 
Assés tenra bien mes conmans,

Que jou a tous amans conmans, Fors tant que ja ne löera

A personne ki amera

Qu'en .I. soel lieu si son coer mete

Que de legier ne s'en demete,

Et vaura as amans aprendre

Conment il devront les las tendre

Por prendre autrui sans estre pris.

(Tou, f. 96bc)

D'ailleurs, pourquoi faut-il s'attendre à une cohérence absolue de la part d'un auteur médiéval? On sait, par exemple, qu'un « clerc » comme Froissart a écrit des œuvres centrées sur l'amour profane. À ce sujet, rappelons quelques mots de Van Hamel à propos de Gilles li Muisis, célèbre abbé de l'abbaye Saint-Martin de Tournai 49 : « il est assez curieux que Gille le Muisit associe presque toujours le Renclus [de Molliens] aux deux auteurs du Roman de la Rose, comme si c'étaient là des ouvrages de même nature " so.

Si l'appartenance du remanieur au clergé (nous utilisons ce terme dans son sens le plus large) est très probable ${ }^{51}$, et si l'état de régulier reste une hypothèse possible, on ne peut en dire autant de son appartenance à un

49. Pour un aperçu de la vie de Gilles li Muisis, voir A. d'Haenens, L'Abbaye Saint-Martin de Tournai de 1290 à 1350. Origines, évolution et dénouement d'une crise, Louvain, 1961 [Recueil de travaux d'histoire et de philologie, IV 23].

50. A.-G. Van Hamel éd., Li Romans de Carité et Miserere du Renclus de Moiliens. Poèmes de la fin du XII siècle, 2 vol., Paris, 1885 [Bibl. de l'École pratique des Hautes Études. Sciences philologiques et historiques, 61-62], t. 1, p. CCI, n. 2. Sur Gilles li Muisis et le Roman de la Rose, voir aussi P.-Y. Badel, Le Roman de la Rose au XIV siècle. Étude de la réception de l'auvre, Genève, 1980 [Publications romanes et françaises, 153], p. 74-82.

51. Voir encore les vers suivants : "Mais maintes fois, en lieu de saumes, / Li verra on ce roumans [le Roman de la Rose] lire " (f. 96c). " En lieu de " peut avoir deux significations : à la place des psaumes, qu'un religieux serait censé lire, mais aussi, plus généralement, comme si c'étaient des psaumes. En revanche, nous ne croyons pas que la place réservée à l'amour des nonnes dans une interpolation qui suit le v. 14398 (Tou f. 121d-122b) ait nécessairement «a particular relevance for clerical readers " (S. Huot, The 'Romance of the Rose' and its Medieval Readers, op. cit., p. 118) : comme l'a fait remarquer F. Lecoy à propos de quelques vers sur le sujet dans le Roman de la Rose (Guillaume de Lorris et Jean de Meun 'Le Roman de la Rose', éd. cit., t. 2, p. 296, n. 14390-14398), « il est souvent question des amours de nonne chez les moralistes médiévaux "; le savant étaie son affirmation à l'aide de nombreuses références, des Carmina Cantabrigensia à Rutebeuf, de Drouart la Vache à Gilles li Muisis, pour n'en citer que quelques-unes. 
ordre mendiant ${ }^{52}$. Il est vrai qu'on aperçoit un certain intérêt pour la querelle qui divisa les ordres mendiants et les séculiers au XIII ${ }^{\mathrm{e}}$ siècle dans le remaniement, comme d'ailleurs dans le Roman de la Rose. Dans le discours de Faux Semblant, le remanieur ajoute trois longues interpolations sur le sujet, une première après le v. 11128 (Tou f. 100c-101b), une deuxième après le v. 11376 (Tou f. 104b-105d) et une troisième après le v. 11568 (Tou f. 106b-107b). Par la voix de Faux Semblant, l'auteur semble justifier la possession de biens et la mendicité pour les Franciscains et les Dominicains, mais il ne faut pas oublier qu'il écrit après les bulles papales Exiit qui seminat, de 1279 s3, et Ad fructus uberes, de 1281-1282 54, qui tranchaient la question en faveur des ordres mendiants.

Cependant, on trouve dans le remaniement quelques passages où l'auteur critique, implicitement, les fautes souvent reprochées aux membres des ordres mendiants, comme dans les vers suivants ${ }^{55}$ :

\author{
Eglise, pour ce t'en casti, \\ Que nous avons .I. plait basti, \\ Dont pluisour seront esmeü \\ Et maint en seront decheü, \\ S'il croient chou que nous dirons ; \\ Mais puis c'a l'avoir partirons, \\ Cui caut que les ames devienent? \\ (Tou, f. 101a) \\ Car se les comfiesses n'estoient, \\ Nos rentes moult petit vauroient. \\ (ibid., f. 106b) \\ Car, puis que je doi dire voir, \\ Je quidai moult bien decevoir \\ Le pape, mais il me dechut, \\ Quant ma petition rechut \\ Et il vit a coy je baoie, \\ Car a nule riens ne tendoie
}

52. Cf. L. Walters, " Gui de Mori's Rewriting of Faux Semblant in the Tournai Roman de la Rose », art. cit.

53. Voir J. H. Sbaralea, Bullarium franciscanum Romanorum pontificum constitutiones, epistolas ac diplomata continens, éd. cit., p. 404d-416d. La bulle à été émise par le pape Nicolas III, et en effet le «papes Nicoles » est nommé deux fois dans l'interpolation qui suit le v. 11376.

54. Voir supra.

55. C'est Faux Semblant qui parle, avec une aporie déjà évidente chez Jean de Meun : cf. K. Brownlee, « The Problem of Faux Semblant : Language, History, and Truth in the Roman de la Rose", dans The New Medievalism, M. S. Brownlee, K. Brownlee et S. G. Nichols éd., Baltimore-Londres, 1991, p. 253-271. 
Fors a ce que, se je peüsce, Simplement son pooir eüsce...

(ibid., f. 106bc)

Saciés, puis que je me conneuch Et que de parler le don euch Et congiet de moi pourcachier, Ains puis ne finai fors trachier Conment je venisse a houneur ; Et pour çou trestous ceaus j'onneur, Et volentiers siu lor compaingne, Qui soeffrent que j'ensi me faingne. Que quels que dedens le coer soie, Je moustre ciere simple et coie, Par coi ceaus puisce decevoir, Dont mon desir voel rechevoir.

(ibid., f. 109b)

En outre, il arrive qu'on trouve dans le remaniement des jugements positifs à propos du clergé séculier, y compris des maîtres de l'Université :

Par pöesté prendent li maistre,

Qui doivent sainte Eglise paistre

Par sainte predication,

Et par lor exortation

Li purgier et enluminer

Et en bon estat affinner,

Que sains Denis claime parfaire ${ }^{56}$,

Ensi conme cil doivent faire

Qui labeurent en sainte Eglise

En aucun publiiet servise ;

Cist poeent bien de l'autrui prendre

Lor soustenance sans mesprendre...

$($ Tou, f. $104 c)$

56. Gui de Mori fait-il allusion ici à la Hiérarchie ecclésiastique du Pseudo-Denys l'Aréopagite, V, I, 6=505C-508D ? Ce n'est pas sûr. Des versions latines des œuvres du Pseudo-Denys étaient bien connues en Europe occidentale au Moyen Âge : cf. H.-F. Dondaine, Le Corpus dionysien de l'Université de Paris au XIII' siècle, Rome, 1953. Voir la traduction de Maurice de Gandillac du passage en question : «...Ainsi l'ordre des ministres est purificateur, puisqu'il élève les purifiés de manière à les rendre dignes de l'illumination des liturgies sacerdotales ; puisqu'il libère les imparfaits de toutes leurs souillures ; puisque, tel un accoucheur, il fait naître en eux les lumières et les leçons purifiantes de l'Écriture " (CEuvres complètes du Pseudo-Denys l'Aréopagite, [Paris], 1990 [1943], 508B, p. 299). Le remanieur cite souvent des auctoritates religieuses, surtout dans le discours de Raison (voir S. Huot, The 'Romance of the Rose' and its Medieval Readers, op. cit., p. 97), mais aussi ailleurs. 
Car une foys l'an au mains doivent

Tout a chelui dont il reçoivent

Sacrement et conmunion

Faire entiere comfiession :

C'est assés raisons et droiture

Que cius ki avra de toi cure

Doie savoir tous les peciés

Simplement dont t'iés enteciés,

Ou de pensees ou de fait ;

Quiconques ensi ne le fait,

Il s'oblige a mort pardurable,

S'il n'a cause tres raisonnable.

(ibid., f. 106d)

L'attitude du remanieur, en somme, est assez équidistante des deux parties, nous semble-t-il, et son intérêt pour la querelle peut s'expliquer simplement par son appartenance au clergé, séculier ou régulier. Son impartialité, dans l'ensemble, vis-à-vis des séculiers et des ordres mendiants ne serait-t-elle pas plutôt l'indice de son appartenance à un autre ordre ? On ne saurait l'affirmer avec certitude.

Nous croyons, enfin, qu'on ne peut rien tirer de concret du fait que, dans le manuscrit de Tournai, à l'intérieur de la lettre $T$ qui ouvre le prologue général, on voit la représentation d'un moine vêtu de blanc, ayant baissé son scapulaire, assis sur un fauteuil doré devant un pupitre, en train d'écrire (f. 5a). Puisque cette miniature est précédée par la rubrique « Chi conmence li prologes que dant Gui de Mori fist sur le Livre de la Rose», nous pensons que l'enlumineur voulait représenter Gui de Mori plutôt que Guillaume de Lorris, comme l'affirmait Lucien Fourez ${ }^{57}$. Mais, tout d'abord, la représentation des auteurs du Roman de la Rose en moines était courante 58 ; à propos de ces représentations, David Hult demande de façon rhétorique : " is this the author writing his own text or the scribe copying the work? ", et peu après il ajoute : " the figure writing in a book is a way of giving a visual, figurative, and fundamentally unrealistic image of the authorial imaginative faculty $"{ }^{59}$. Deuxièmement, nous avons déjà dit que rien ne prouve que Tou soit un manuscrit contrôlé par l'auteur ${ }^{60}$.

57. "Le Roman de la Rose de la Bibliothèque de la Ville de Tournai », dans Scriptorium, t. 1 (1946-1947), p. 213-239, pl. 21-24, à la p. 220, n. 17.

58. Cf. D. Hult, Self-fulfilling Prophecies. Readership and Authority in the First 'Roman de la Rose', Cambridge, 1986, p. 82, et les reproductions aux p. 80 et 85-88.

59. Ibid., p. $78-79$ et 80 .

60. Deux mains connues travaillèrent aux enluminures de Tou, celle du maître du cérémonial de Gand et celle d'un jeune Pierart dou Tielt, plus une troisième non identifiée (voir F. Avril, « Manuscrits », dans Les Fastes du Gothique. Le siècle de Charles $V$, catalogue de l'exposition, Galeries nationales du Grand Palais, 9 octobre 
Certes, les enlumineurs médiévaux cherchaient souvent à indiquer la position sociale des auteurs avec précision, quand ils la connaissaient ${ }^{61}$, et naturellement les enlumineurs de Tou pouvaient être mieux renseignés que nous à l'égard de Gui de Mori : dans ce cas, le choix de le représenter en moine, et en moine vêtu de blanc, pourrait ne pas être fortuit. Le blanc était notamment la couleur de l'habit des cisterciens ${ }^{62}$; les dominicains aussi portaient des costumes blancs, mais leurs capes étaient noires et, de plus, on vient de voir la difficulté de pouvoir considérer Gui de Mori comme appartenant à un ordre mendiant (Faux Semblant en revanche est clairement représenté en dominicain au f. $98 \mathrm{v}$ de Tou). L'appartenance de Gui de Mori à l'ordre cistercien ne pose pas problème en soi, mais une telle affirmation nous paraît, en l'état, manquer de fondements scientifiques.

En conclusion, l'analyse textuelle et le commentaire de la morphologie et de la syntaxe ayant permis de montrer que le remaniement date de 1290 , son auteur ne peut être identifié avec Guibert de Tournai, mort en 1284 : nous nous contentons donc d'appeler l'auteur par le nom qu'il se donne lui-même, implicitement dans la devinette et explicitement dans le discours du dieu d'amour, Gui(s) de Mo(i)ri. On peut affirmer qu'il était d'origine picarde, comme le prouve sa langue; certaines allusions dans le texte, enfin, semblent suggérer qu'il appartenait au clergé, peut-être au clergé régulier.

Andrea VALENTini.

1981-1 ${ }^{\text {er }}$ février 1982, Paris, 1981, p. 276-362, aux p. 301-303). La miniature en question serait due au maître du cérémonial ou à l'autre enlumineur (ibid., p. 301).

61. Cf. M. L. Meneghetti, Il pubblico dei trovatori. La ricezione della poesia cortese fino al XIV secolo, Turin, 1992 2 , notamment aux p. 245-264.

62. Sur la couleur de l'habit des cisterciens, voir un ouvrage récent : La sostanza dell'effimero. Gli abiti degli ordini religiosi in Occidente, G. Rocca éd., catalogue de l'exposition, Museo nazionale di Castel Sant'Angelo, 18 janvier-31 mars 2000, Rome, 2000 , p. 165-166, renvois bibliographiques à la p. 168. À noter que Guillaume de Lorris utilise l'expression «blanc moine » dans le Roman de la Rose, au v. 3034 (Langlois v. 3050); elle est glosée « Cisterciens " par Langlois (Guillaume de Lorris et Jean de Meun 'Le Roman de la Rose', éd. cit., t. 2, p. 323). 